Conclusions These results support the use of NCV delivered by DNA-EP with $\alpha$ CTLA-4 and suggest a new combined therapy for clinical testing.

Disclosure Information F. Palombo: A. Employment (full or part-time); Significant; Neomatrix biotech. E. Salvatori: A. Employment (full or part-time); Significant; Takis biotech. L. Lione: A. Employment (full or part-time); Significant; Takis biotech. M. Compagnone: A. Employment (full or part-time); Significant; Neomatrix biotech. A. Conforti: A. Employment (full or part-time); Significant; Evvivax. L. Aurisicchio: A. Employment (full or part-time); Significant; Neomatrix biotech, Takis biotech, Evvivax.

\section{P08.04 SUCCESSFUL IMMUNOTHERAPY OF THE BREAST CANCER METASTATIC DISEASE IN MICE USING A PHARMACEUTICAL TLR4-AGONIST INDUCES SYSTEMIC ANTI-TUMOR T CELL RESPONSE AND LONG-TERM T CELL MEMORY}

E Ushakova*, E Lebedeva, A Pichugin, R Ataullakhanov. NRC Institute of Immunology FMBA of Russia, Moscow, Russian Federation

\subsection{6/jitc-2021-ITOC8.48}

Background A study of the anti-tumor T-cell response and immunological memory following successful 4T1 breast cancer immunotherapy with the combination of surgical resection of the primary tumor and subsequent macrophage/dendritic cell reprogramming using injections of the pharmaceutical TLR4agonist.

Materials and Methods 15,000 cells of the 4T1 mouse breast carcinoma inoculated subcutaneously into BALB/c mice generated solid tumors and metastatic disease ended by the death of all the tumor-bearing animals during 30-40 days. Surgical resection of the primary tumor was performed on day 11 . Pharmaceutical TLR4-agonist $\left(\operatorname{Immunomax}{ }^{\circledR}\right.$ ) administered intraperitoneally in dose of $14 \mu \mathrm{g}$ every 2-3 days, in total seven injections per course. Sorted macrophage/dendritic cells reprogramming was examined by RT-PCR. Tumor-reactive IFN $\gamma$-secretory $\mathrm{T}$ cells were counted using ELISPOT in ex vivo co-cultures of sorted CD4 $\mathrm{T}$ cells or CD8 $\mathrm{T}$ cells with the tumor lysate-loaded syngeneic dendritic cells or alive 4T1 tumor cells. Sorted CD8 effector T cell cytotoxicity was measured in their co-culture with different numbers of 4T1 target cells.

Results Using a combination of surgical resection of the primary 4T1 tumor and immunotherapy with the pharmaceutical TLR4-agonist for the treatment of metastatic disease in $\mathrm{BALB} / \mathrm{c}$ mice a complete recovery of $20-30 \%$ mice was achieved. The complete responder mice effectively generated CD4 T cells and CD8 T cells, which specifically respond to 4T1 tumor antigens by IFN-production and kill 4T1 tumor cells in ex vivo co-cultures. The T-cell response is systemic, as tumor-specific $\mathrm{T}$ cells accumulate in the spleen. The second or third inoculation of the 4T1 tumor is accompanied by a complete absence of tumor growth in 50\% and inhibition of tumor growth in the rest of the immune mice. An accumulation of significant numbers of $\mathrm{T}$ cells that respond to 4T1 tumor antigens by IFN $\gamma$-secretion, as well as of CD8 $\mathrm{T}$ cells that kill 4T1 tumor cells in a cytotoxic test was found in the secondary (tertiary) tumors, as well as in the draining lymph nodes. Immunological memory in complete responder mice that recovered due to the treatment with resection of the primary tumor and immunotherapy with a 4T1-agonist persisted for a long time (maximum observation period of 260 days).

Conclusions Macrophage/dendritic cell reprogramming with the TLR4-agonist for the post-resectional immunotherapy of 4T1 breast cancer metastatic disease induce tumor-specific CD4 and CD8 T cell responses and T-cell mediated long-living immune memory.

Funding This study was supported by the Russian Science Foundation (project no. 20-15-00391).

Disclosure Information E. Ushakova: None. E. Lebedeva: None. A. Pichugin: None. R. Ataullakhanov: None.

\section{P08.05 COMBINED PHARMACOLOGICAL TARGETING OF ADENOSINE 2A- AND 2B-RECEPTOR ENHANCES CAR T CELL FUNCTION}

'M Seifert*, M Benmebarek ', B Cadilha ${ }^{1}$, '1 Jobst, 'J Dörr, 'T Lorenzini, 'D Dhogina, ${ }^{1} \mathrm{~J}$ Zhang, ${ }^{1} \mathrm{~J}$ Zhang, ${ }^{2} \mathrm{U}$ Schindler, ${ }^{1} \mathrm{~S}$ Endres, ${ }^{1,3} \mathrm{~S}$ Kobold. ${ }^{1}$ Division of Clinical Pharmacology, Department of Medicine IV, University Hospital, Ludwig Maximilian, Munich, Germany; ${ }^{2}$ Independent scientist with past affiliation Arcus Biosciences, Inc., 3928 Point Eden Way, Hayward, CA, USA; ${ }^{3}$ German Center for Translational Cancer Research (DKTK), partner site Munich, Germany

\subsection{6/jitc-2021-ITOC8.49}

Background Despite remarkable response rates mediated by anti-CD19 chimeric antigen receptor (CAR) $\mathrm{T}$ cells in selected $\mathrm{B}$ cell malignancies, CAR $\mathrm{T}$ cell therapy still lacks efficacy in the vast majority of tumors. A substantial limiting factor of CAR $\mathrm{T}$ cell function is the immunosuppressive tumor microenvironment. Among other mechanisms, the accumulation of adenosine within the tumor can contribute to disease progression by suppressing anti-tumor immune responses. Adenosine $2 \mathrm{a}$ - and $2 \mathrm{~b}$-receptor $\left(\mathrm{A} 2_{\mathrm{A}}\right.$ and $\left.\mathrm{A} 2_{\mathrm{B}}\right)$-mediated cAMP build-up suppresses $\mathrm{T}$ cell effector functions. In the present study we hypothesize, that combination therapy with the selective $\mathrm{A} 2 \mathrm{~A} /$ $\mathrm{A} 22_{\mathrm{B}}$ dual antagonist AB928 (etrumadenant) enhances CAR T cell efficacy.

Materials and Methods Second generation murine (antiEPCAM) and human (anti-MSLN) CAR constructs, containing intracellular CD28 and CD3 $\zeta$ domains, were fused via overlap extension PCR cloning. Murine or human T cells were retrovirally transduced to stably express the CAR constructs. $\mathrm{A} 2 \mathrm{~A}$ $\mathrm{A} 2 \mathrm{~B}_{\mathrm{B}}$ signaling in CAR $\mathrm{T}$ cells was analyzed by phospho-specific flow cytometry of CREB (pS133)/ATF-1 (pS63). CAR T cell activation was quantified by flow cytometry and enzyme-linked immunosorbent assay (ELISA) of IFN- $\gamma$, IL- 2 and TNF- $\alpha$. CAR $\mathrm{T}$ cell proliferation was assessed by flow cytometry. CAR $\mathrm{T}$ cell cytotoxicity was assessed by impedance based real-time cell analysis.

Results AB928 protected murine CAR $\mathrm{T}$ cells from cAMP response element-binding protein (CREB) phosphorylation in the presence of stable adenosine analogue 5'-N-ethylcarboxamidoadenosine (NECA). NECA inhibited antigen-dependent CAR $T$ cell cytokine secretion in response to four murine tumor cell lines. CAR T cell-mediated tumor cell lysis as well as proliferation were decreased in the presence of NECA or adenosine. Importantly, AB928 fully restored CAR T cell cytotoxicity, proliferation, and cytokine secretion in a dose dependent manner. Further, AB928 also restored antigen dependent cytokine secretion of human CAR $\mathrm{T}$ cells in the presence of NECA. 